\title{
Split Operator Methods for Hyperbolic Systems in $p$ Space Variables
}

\author{
By A. R. Gourlay and A. R. Mitchell
}

Introduction. In recent papers [2], [3], the present authors introduced two alternating direction procedures for the numerical solution of the first-order hyperbolic system

$$
U_{t}=A U_{x}+B U_{y},
$$

where $U$ is an $n$-component vector function of the space co-ordinates $x, y$ and the time $t$, and $A, B$ are $n \times n$ symmetric matrices which may depend on $x, y$ and $t$. In this note it will be shown how these methods may be extended to the $p$ spacedimensional system

$$
U_{t}=\sum_{i=1}^{p} A_{i} U_{x_{i}}
$$

where $A_{i}(i=1, \cdots p)$ are $n \times n$ symmetric matrices and $U$ is an $n$-component vector function of the space co-ordinates $x_{i}(i=1, \cdots p)$ and $t$. In Sections 1 and 2 it will be assumed that the matrices are independent of $x_{i}(i=1, \cdots p)$ and $t$. The variable coefficient case will be treated in Section 3 .

1. Two Space Dimensions. An example of the first type of alternating direction procedure for the numerical solution of (1) [2], is given by

$$
\begin{aligned}
& {\left[I+\frac{1}{2}(I-r B) \Delta_{y}\right] U_{m+1}^{(1)}=\left[I+\frac{1}{2}(I+r A) \Delta_{x}\right] U_{m},} \\
& {\left[I+\frac{1}{2}(I-r A) \Delta_{x}\right] U_{m+1}=\left[I+\frac{1}{2}(I+r B) \Delta_{y}\right] U_{m+1}^{(1)},}
\end{aligned}
$$

where $U_{m}, U_{m+1}$ are the values of $U$ at the mesh points $(i h, j h, m k)$ and $(i h, j h$, $(m+1) k)$ respectively, $I$ is the unit matrix of order $n$, and $r=k / h$, where $h, k$ are the mesh increments in the space and time directions respectively. The quantity $U_{m+1}^{(1)}$ is an auxiliary solution and in general is not an approximation to the solution of (1) at any time. The forward-difference operators $\Delta_{x}, \Delta_{y}$ are defined in the usual manner. The elimination of $U_{m+1}^{(1)}$ from the Eqs. (2) gives

$$
\begin{aligned}
{\left[I+\frac{1}{2}(I-r B) \Delta_{y}\right]\left[I+\frac{1}{2}(I-r A) \Delta_{x}\right] U_{m+1} } \\
\left.=\left[I+\frac{1}{2}(I+r B) \Delta_{y}\right]_{L} I+\frac{1}{2}(I+r A) \Delta_{x}\right] U_{m} .
\end{aligned}
$$

The formulae (2) and (3) constitute the split and factorised forms of one of a family of sixteen similar methods (see [2]).

The second alternating direction procedure [3] is given by

$$
\begin{aligned}
& {\left[I-\frac{1}{4} r B\left(\Delta_{y}+\nabla_{y}\right)\right] U_{m+1}^{(1)}=\left[I+\frac{1}{4} r A\left(\Delta_{x}+\nabla_{x}\right)\right] U_{m},} \\
& {\left[I-\frac{1}{4} r A\left(\Delta_{x}+\nabla_{x}\right)\right] U_{m+1}=\left[I+\frac{1}{4} r B\left(\Delta_{y}+\nabla_{y}\right)\right] U_{m+1}^{(1)},}
\end{aligned}
$$

Received June 22, 1966. 
where $\nabla_{x}, \nabla_{y}$ are the first-order backward-difference operators in the $x$ and $y$ directions respectively. The elimination of $U_{m+1}^{(1)}$ from (4) leads to the scheme

$$
\begin{aligned}
& {\left[I-\frac{1}{4} r B\left(\Delta_{y}+\nabla_{y}\right)\right]\left[I-\frac{1}{4} r A\left(\Delta_{x}+\nabla_{x}\right)\right] U_{m+1}} \\
& =\left[I+\frac{1}{4} r B\left(\Delta_{y}+\nabla_{y}\right)\right]\left[I+\frac{1}{4} r A\left(\Delta_{x}+\nabla_{x}\right)\right] U_{m} .
\end{aligned}
$$

2. Extension to the Case of $p$ Space Variables. Let us now consider how the methods described above may be extended to the case of $p(>2)$ space variables. The system of differential equations to be considered is given by

$$
U_{t}=\sum_{i=1}^{p} A_{i} U_{x_{i}}
$$

where $A_{i}(i=1, \cdots p)$ are symmetric $n \times n$ matrices.

The natural extension of (3) to $p$ space variables is given by

$$
\prod_{s=1}^{p}\left[I+\frac{1}{2}\left(I-r A_{s}\right) \Delta_{x_{s}}\right] U_{m+1}=\prod_{s=1}^{p}\left[I+\frac{1}{2}\left(I+r A_{s}\right) \Delta_{x_{s}}\right] U_{m} .
$$

where $U_{m}=U\left(i_{1} h, \cdots, i_{s} h ; m k\right), U_{m+1}=U\left(i_{1} h, \cdots, i_{s} h ;(m+1) k\right)$, and the order of the matrices in the product is defined by

$$
\prod_{s=1}^{p} Q_{i}=Q_{1} Q_{2} \cdots Q_{p}
$$

The formula (7) connects the values of $U$ at $2^{p}$ points at each of two neighbouring time levels. In a similar manner (5) may be extended to give

$$
\prod_{s=1}^{p}\left[I-\frac{1}{4} r A_{s}\left(\Delta_{x_{s}}+\nabla_{x_{s}}\right] U_{m+1}=\prod_{s=1}^{p}\left[I+\frac{1}{4} r A_{s}\left(\Delta_{x_{s}}+\nabla_{x_{s}}\right)\right] U_{m},\right.
$$

a formula involving $3^{p}$ points at each of two adjacent time levels.

The above formulae, which are correct to $O\left(h^{2}\right)$, are of the form

$$
\prod_{s=1}^{p} B_{s} U_{m+1}=\prod_{s=1}^{p} C_{s} U_{m}
$$

where $B_{s}, C_{s}$ take the values

$$
I+\frac{1}{2}\left(I \mp r A_{s}\right) \Delta_{x_{s}}
$$

or

$$
I \mp \frac{1}{4} r A_{s}\left(\Delta_{x_{s}}+\nabla_{x_{s}}\right)
$$

depending on whether we are referring to method (7) or (8).

The obvious generalization of the split formulae (2) and (4) to $p(>2)$ space dimensions is

$$
\begin{aligned}
B_{p} U_{m+1}^{(1)} & =C_{1} U_{m}, \\
B_{p+1-q} U_{m+1}^{(q)} & =C_{q} U_{m+1}^{(q-1)}, \quad(q=2, \cdots, p-1) \\
B_{1} U_{m+1} & =C_{p} U_{m+1}^{(p-1)},
\end{aligned}
$$

where $U_{m+1}^{(q)},(q=1, \cdots)$, are intermediate values. Unfortunately this splitting 
requires that the matrices $A_{2}, \cdots, A_{p}$ commute. It is unlikely that this will occur in practice and so this Peaceman-Rachford type factorization for the case of $p(>2)$ space variables is of little value. It may be verified in a similar manner that the above commutation conditions are also required for Douglas-Rachford type factorizations. Moreover, any attempt to extend to $p(>2)$ space variables the boundary procedures advocated in [2] and [3] for the schemes (2) and (4) has resulted in failure.

We therefore require a splitting of (9) which does not demand any commutation of the matrices $A_{i}(i=1,2, \cdots, p)$ and admits easy incorporation of boundary data at intermediate steps. Such a splitting is given by $\mathrm{D}^{\prime} \mathrm{Jakonov}$ [1]. For (9) this becomes

$$
\begin{aligned}
& B_{1} U_{m+1}^{(1)}=\prod_{s=1}^{p} C_{s} U_{m}, \\
& B_{q} U_{m+1}^{(q)}=U_{m+1}^{(q-1)}, \quad(q=2, \cdots p)
\end{aligned}
$$

where $U_{m+1}^{(p)}=U_{m+1}$ and the "boundary" values $\widetilde{U}_{m+1}^{(q)}$ at the intermediate levels are obtained from the formulae

$$
\widetilde{U}_{m+1}^{(q)}=\prod_{s=q+1}^{p} B_{s} \widetilde{U}_{m+1}, \quad q=1,2, \cdots,(p-1) .
$$

Thus the methods of [2], [3] may be extended to $p(>2)$ space dimensions, provided we employ a $\mathrm{D}^{\prime}$ Jakonov type splitting.

3. Variable Coefficients. If the matrices $A_{i}(i=1, \cdots, p)$ are functions of the space variables $x_{i}(i=1, \cdots, p)$ and $t$, it can be verified by the Taylor expansions that the difference formula (8) is still accurate to second order provided the matrix operators $B_{s}, C_{s}$ are evaluated at the $(m+1)$ th and $m$ th time levels respectively. However, in the case of formula (7), the matrix operators must not only be evaluated at the correct time level but also at the center of the hypercube formed by the $2^{p}$ points at each time level. This centering in space was first employed by Wendroff [5] for his two-level, $2 \times 2^{p}$ point scheme.

4. Stability. If we carry out a Fourier transformation of the space variables in the usual way, (9) becomes

$$
\prod_{s=1}^{p} b_{s} u^{(m+1)}=\prod_{s=1}^{p} c_{s} u^{(m)}
$$

where $b_{s}, c_{s}$ take the values

$$
I \cos \left(\beta_{s} h / 2\right) \mp i r A_{s} \sin \left(\beta_{s} h / 2\right)
$$

or

$$
I \mp \frac{1}{2} i r A_{s} \sin \beta_{s} h,
$$

depending on whether we are considering method (7) or method (8). The quantities $\beta_{s}(s=1, \cdots p)$ are arbitrary real numbers. The local amplification matrix of $(9)$ is then given by 


$$
G=\left(\prod_{s=1}^{p} b_{s}\right)^{-1}\left(\prod_{s=1}^{p} c_{s}\right) .
$$

Applying the stability analysis outlined in [3], it can be shown for methods $(7)$ and (8) that

$$
\left\|G^{*} G\right\| \leqslant 1+O\left(k^{2}\right)
$$

where $G^{*}$ is the complex conjugate of $G$, and where \|\| denotes the $L_{2}$ norm. Since a necessary and sufficient condition for stability (Lax and Richtmyer [4]) is given by

$$
\left\|G^{*} G\right\| \leqslant 1+O(k)
$$

it follows that methods (7) and (8) are unconditionally stable. If the matrices $A_{s}(s=1, \cdots p)$ commute, then (12) reduces to

$$
\left\|G^{*} G\right\|=1 .
$$

In [3] it was shown, for the case $p=2$, that the commutation condition is not necessary for the best constant one to appear on the right-hand side of (13).

Acknowledgement. Mr. A. R. Gourlay's share of the work was carried out whilst he was in receipt of a Carnegie Research Scholarship.

University of St. Andrews

St. Andrews, Scotland

1. E. G. D'JAKonov, "Difference schemes with a disintegrating operator for multidimensional problems," Ż. Vyčisl. Mat. i Mat. Fiz., v. 4, 1963, pp. 581-607. (Russian)

2. A. R. Gourlay \& A. R. Mitchell, "Alternating direction methods for hyperbolic systems," Numer. Math., v. 8, 1966, pp. 137-149.

3. A. R. GouRLAY \& A. R. Mitchell, "A stable implicit method for hyperbolic systems in two space dimension," Numer. Math., v. 8, 1966, pp. 367-375.

4. P. D. Lax \& R. D. Richtmyer, "Survey of the stability of linear finite difference equations," Comm. Pure Appl. Math., v. 9, 1956, pp. 267-293. MR 18, 48.

5. B. WENDROFF, "On centered difference equations for hyperbolic systems, " $J$. Soc. Indust. A ppl. Math., v. 8, 1960, pp. 549-555. MR $22 \# 7259$. 